\title{
Adaptive microfluidic gradient generator for quantitative chemotaxis experiments
}

Alexander Anielski, Eva K. B. Pfannes, and Carsten Beta

Citation: Review of Scientific Instruments 88, 034301 (2017); doi: 10.1063/1.4978535

View online: http://dx.doi.org/10.1063/1.4978535

View Table of Contents: http://aip.scitation.org/toc/rsi/88/3

Published by the American Institute of Physics

\section{Articles you may be interested in}

In situ and time-resolved infrared detection of the reactivity induced by electrons in polymer films

Review of Scientific Instruments 88, 034102034102 (2017); 10.1063/1.4978442

A high precision gas flow cell for performing in situ neutron studies of local atomic structure in catalytic materials

Review of Scientific Instruments 88, 034101034101 (2017); 10.1063/1.4978287

Chemical aerosol Raman detector

Review of Scientific Instruments 88, 033107033107 (2017); 10.1063/1.4977725

Fast, precise, and widely tunable frequency control of an optical parametric oscillator referenced to a frequency comb

Review of Scientific Instruments 88, 033101033101 (2017); 10.1063/1.4977049

An in situ trap capacitance measurement and ion-trapping detection scheme for a Penning ion trap facility

Review of Scientific Instruments 88, 034705034705 (2017); 10.1063/1.4978799

A hybrid pulse combining topology utilizing the combination of modularized avalanche transistor Marx circuits, direct pulse adding, and transmission line transformer

Review of Scientific Instruments 88, 033507033507 (2017); 10.1063/1.4978650

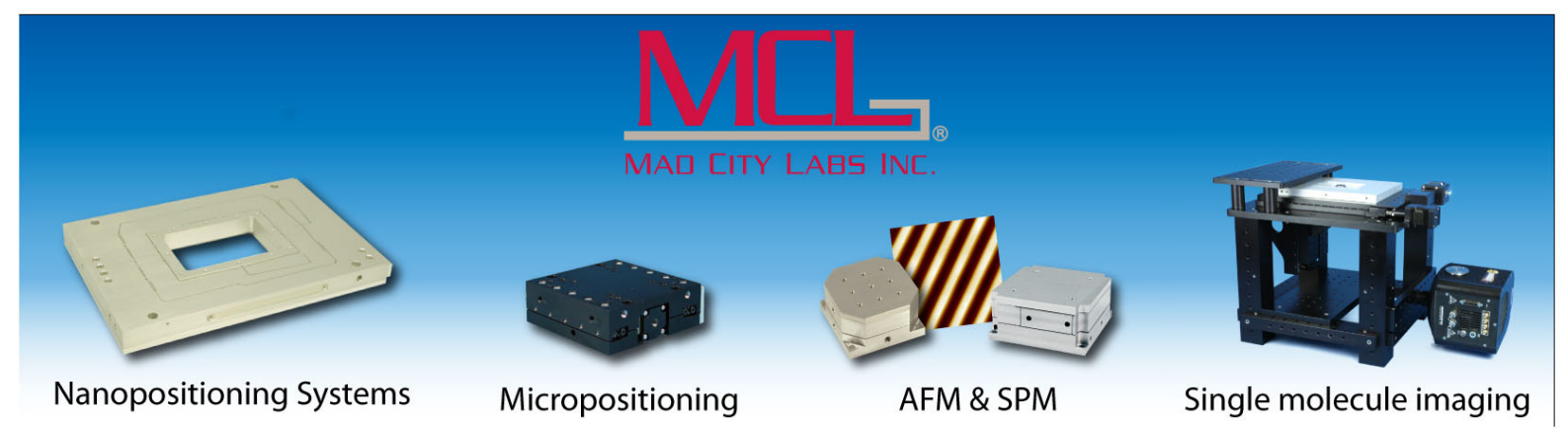




\title{
Adaptive microfluidic gradient generator for quantitative chemotaxis experiments
}

\author{
Alexander Anielski, Eva K. B. Pfannes, ${ }^{\text {a) }}$ and Carsten Betab) \\ Institute of Physics and Astronomy, University of Potsdam, Karl-Liebknecht-Str. 24/25, 14476 Potsdam, Germany
}

(Received 14 November 2016; accepted 28 February 2017; published online 22 March 2017)

\begin{abstract}
Chemotactic motion in a chemical gradient is an essential cellular function that controls many processes in the living world. For a better understanding and more detailed modelling of the underlying mechanisms of chemotaxis, quantitative investigations in controlled environments are needed. We developed a setup that allows us to separately address the dependencies of the chemotactic motion on the average background concentration and on the gradient steepness of the chemoattractant. In particular, both the background concentration and the gradient steepness can be kept constant at the position of the cell while it moves along in the gradient direction. This is achieved by generating a well-defined chemoattractant gradient using flow photolysis. In this approach, the chemoattractant is released by a light-induced reaction from a caged precursor in a microfluidic flow chamber upstream of the cell. The flow photolysis approach is combined with an automated real-time cell tracker that determines changes in the cell position and triggers movement of the microscope stage such that the cell motion is compensated and the cell remains at the same position in the gradient profile. The gradient profile can be either determined experimentally using a caged fluorescent dye or may be alternatively determined by numerical solutions of the corresponding physical model. To demonstrate the function of this adaptive microfluidic gradient generator, we compare the chemotactic motion of Dictyostelium discoideum cells in a static gradient and in a gradient that adapts to the position of the moving cell. Published by AIP Publishing. [http://dx.doi.org/10.1063/1.4978535]
\end{abstract}

\section{INTRODUCTION}

Chemotaxis, the directed motion of a cell towards a source of chemoattractant, plays a key role in many biological processes including morphogenesis, wound healing, and cancer metastasis. ${ }^{1}$ Laboratory investigations of chemotaxis require techniques to generate a chemoattractant concentration gradient. Over the past decades, a wide repertoire of gradient generating techniques has been established. ${ }^{2}$ One of the simplest methods is the micropipette assay based on the diffusion of the chemoattractant out of a glass capillary. It generates a radially symmetric gradient profile that allows for rapid qualitative tests but is not suited for quantitative longtime measurements under defined conditions as the concentration profile is not well controlled and varies with time.

More elaborate classical gradient assays rely on diffusion to establish a linear gradient between two reservoirs of liquid with different chemoattractant concentrations. For example, the Dunn chamber consists of a glass slide with two concentric wells that contain different concentrations of chemoattractant. A coverslip with the cells attached to it is placed on top of the glass slide, forming a narrow gap between the two reservoirs where the gradient is formed by diffusion. ${ }^{3}$ Similar designs and variations of it have been proposed ${ }^{4,5}$ and are successfully used to study chemotactic motion until today. 6,7

\footnotetext{
a) Present address: Charité - Universitätsmedizin Berlin, Department of Dermatology and Allergy, Clinical Research Center for Hair and Skin Science, Charitéplatz 1, D - 10117 Berlin, Germany.

b) Author to whom correspondence should be addressed. Electronic mail: beta@uni-potsdam.de
}

Over the past decade, microfluidic techniques have been established as a popular tool to perform live cell experiments in well-controlled micro-environments. ${ }^{8}$ With the advent of polydimethylsiloxane (PDMS)-based soft lithography, well-established low-cost protocols for the routine fabrication of microfluidic chambers became widely available. ${ }^{9}$ Also chemoattractant gradients can be readily generated in a microfluidic device. ${ }^{10-12}$ In many cases, gradients are generated by diffusion between adjacent layers of liquid in a laminar flow. The most prominent example based on this principle is the pyramidal mixing network, where liquid of two different concentrations is pumped through a multi-level bifurcating microchannel network. ${ }^{13,14}$ At each level of the network, liquid from neighboring channels is diffusively mixed to create an intermediate chemoattractant concentration, so that finally multiple equidistant concentration levels between the two initial concentrations are generated and joined together to form a linear gradient profile perpendicular to the flow. Besides gradient mixers that operate under continuous flow conditions, also flow-free microfluidic gradient devices have been designed and are used, for example, to study the motion of chemotactic bacterial swimmers. ${ }^{15}$

While common microfluidic gradient devices offer excellent control over the chemoattractant profile on a micron scale, ${ }^{16}$ the temporal resolution is typically low. In most cases, it takes several minutes or longer to establish a stable gradient profile or to switch its direction. In contrast to this, upstream chemotactic signaling events occur already a few seconds after receptor stimulus. ${ }^{17}$ Detailed studies of the chemotactic signaling dynamics thus require methods to apply chemoattractant stimuli with a temporal resolution that matches this time scale. 
To meet this challenge, we have developed the flow photolysis approach. ${ }^{18}$ Flow photolysis combines the advantages of microfluidic tools with the photochemical release of caged signaling molecules. Cells are placed in a microfluidic channel under a gentle, constantly running fluid flow that contains a biologically inert, caged signaling agent. To stimulate individual cells, the caging group is split off by short wavelength irradiation inside a confined region immediately upstream of the cell. The released substance is then transported by the flow downstream across the cell. Sub-second switching times of chemical stimuli can be achieved in this way. ${ }^{19,20}$ Furthermore, the spatial distribution of the released substance can be precisely controlled. The wider the illuminated region extends in the direction of the flow, the longer is the caged substance exposed to the light source and the more signaling substance will be released. Based on this simple interplay of fluid flow and light source geometry, a wide variety of concentration profiles can be tailored. ${ }^{18,21}$ We can thus control chemical stimuli on the length scales of individual cells with sub-second temporal resolution.

In its original form, chemotaxis experiments based on flow photolysis share a common limitation that is inherent to all gradient generating devices: while the gradient steepness remains constant for a chemotactic cell that moves in a linear gradient, the average background concentration that the cell experiences changes as it moves along the gradient. None of the available gradient techniques provides conditions such that both the gradient steepness and the background concentration remain constant as the cell moves up the gradient. Here we will present an experimental setup that fills this gap. The setup enables us to perform chemotactic migration experiments in the course of which the concentration profile is kept constant at the location of a moving cell. This is achieved by combining the flow photolysis method with a real-time cell tracking algorithm that controls a motorized microscope stage. By moving the stage, the setup compensates the motion of the cell in such a way that it remains at a constant midpoint concentration in the chemoattractant gradient.
Our method is limited to single cell experiments. Simultaneous measurements on populations of cells are not possible. As we rely on flow photolysis, our approach is also limited to chemoattractants that are available in a caged variant. The direct quantification of concentration profiles released by flow photolysis is difficult. The most common way relies on comparison with the release of caged fluorescent dyes. Also, fluorescent cages are available, so that the amount of released signaling substance is directly related to the fluorescence signal. However, due to the small volumina in microfluidic chambers and the minute amounts of released cage, this approach is hampered by very small signal intensities. In this work, we propose an alternative approach that relies on determining the concentration profile by numerical solution of a physical model that includes all relevant processes involved in the release of the caged compound. This allows us to calculate the concentration profile at every time point during the imaging process.

The paper is organized as follows. In Section II, the experimental setup is introduced, including the image processing procedure and the fabrication of microfluidic devices. The theoretical basis of calculating the concentration profile that results from flow photolysis is presented in Section III. Section IV presents results that demonstrate the performance of our setup and provide a first representative study of Dictyostelium chemotaxis under different gradient conditions that can only be achieved with this type of setup. The article closes with a conclusion in Section V.

\section{MATERIALS AND METHODS}

\section{A. Imaging setup}

The imaging setup consists of an Olympus IX 71 microscope (Olympus) with a motorized microscope stage (Scan $112 \times 74$ and Controller Tango2 Desktop 1.25 A, Märzhäuser), see Fig. 1 for a schematic representation of the setup. The position of the stage can be controlled via a serial interface with a precision of $1 \mu \mathrm{m}$. On the stage,

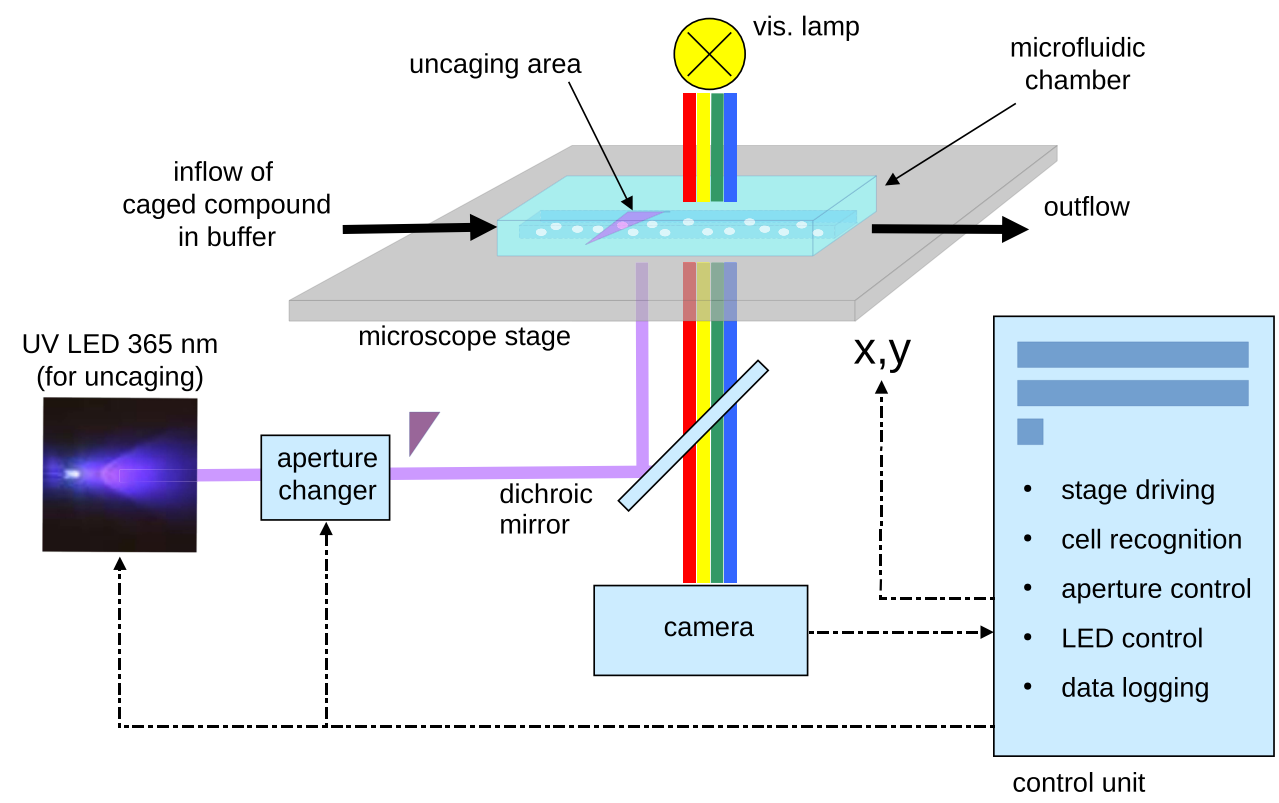

FIG. 1. Schematic presentation of the imaging setup illustrating light path, sample position, and connection to the control unit. 
the microfluidic device is fixed in the optical light path (for fabrication of the device, see Sec. II C). With a LUCPlanFLN 20X/0.45 PH1 phase contrast objective (Olympus) and an Olympus F-View II greyscale camera, phase contrast images of the amoeba cells are recorded.

A M365L2 UV-light-emitting diode (LED) (Thorlabs) emits light with a wavelength of $365 \mathrm{~nm}$ that lies well within the absorption peak of DMNB-caged $\mathrm{AMP}^{22}$ and provides the energy that leads to photolysis of the caged compound. The light emitted by the LED passes a collimator and a filter wheel which carries several apertures of different shapes. The UV beam is coupled into the imaging light path using a U-MWU2 filter block (Olympus) and illuminates an area within the field of view upstream of the cell of interest. In this way, uncaged cAMP molecules are transported from the illuminated area with the fluid flow towards the cells. The differently shaped apertures are used to create different concentration gradient profiles based on the principle of flow photolysis. ${ }^{18}$ The downstream distance between the illuminated area and the cells has to be chosen as small as possible to avoid blurring of the concentration profile by lateral diffusion, but large enough in order to prevent damaging the cells by UV stray light. In our case, the downstream distance was set to approximately $350 \mu \mathrm{m}$.

The spatially resolved UV light density was calculated from a fluorescence image of the microchannel filled with a fluorescein solution and the total power which was measured by an optical power meter (Thorlabs PM100).

\section{B. Image processing and control}

Phase contrast images taken with the Olympus F-View II camera are directly transferred to a computer for further processing with Matlab (MathWorks $\mathrm{GmbH}$ ). To lower the CPU load, realtime image processing is restricted to a rectangular (i.e., $130 \times 115 \mu \mathrm{m}^{2}$ ) region of interest (ROI) within the field of view, which corresponds to a field of about $460 \times 350$ pixel. First, a brightness normalization is carried out by dividing each grayscale value by the global standard deviation. In order to reduce the influence of noise in the subsequent image segmentation process, the images are preprocessed with a Wiener filter. ${ }^{23}$ For contour detection the absolute value of the normalized gradient image is calculated. By thresholding normalized gradient values greater than 1.0, a binary mask was generated. The surface within closed edges was filled with the Matlab function imfill. The Matlab function bwlabel assigns numbers to all detected areas. Our algorithm ${ }^{24}$ then chooses the area of interest based on a weighting function that considers the cell area and its distance from the center of the ROI. Finally, depending on the modus of operation, the position of the ROI or the microscope stage is adjusted in order to center the cell of interest within the ROI. The different modi of operation are described below.

\section{Static spatial gradient}

The cell moves along a chemoattractant gradient that is created by flow photolysis in the direction of increasing concentration. While the cell moves, the average concentration at its location increases (if the gradient profile is not linear, also the gradient steepness may change). This corresponds to the setting typically encountered in natural situations and in most of the common gradient generating devices. In this modus, the algorithm moves the ROI so that the cell stays in its center at every time step.

\section{Dynamically adapted spatial gradient}

The cell moves along a chemoattractant gradient in the direction of increasing concentration. While the cell moves, both the gradient profile and the average concentration remain constant at the location of the cell. This is achieved by compensating cell movement by moving the microscope stage. In this way, the ROI with the cell at its center stays at the same place in the field of view and thus also at the same location in the gradient created by flow photolysis. A further benefit of this modus is that chemotaxis can be recorded over a long time, as the cell cannot leave the field of view and does not reach saturation levels as the surrounding chemoattractant concentration remains constant.

\section{Temporal gradient}

The cell is exposed to a spatially uniform concentration of chemoattractant. This can be achieved by performing flow photolysis with a rectangular aperture in front of the uncaging light source. Again, cell movement is compensated by the movement of the microscope stage, so that the ROI with the cell at its center stays at the same place in the field of view. However, now we couple the direction of cell movement to the intensity of the uncaging light source, so that movement in a given direction is rewarded by an increase of the spatially uniform chemoattractant concentration, while movement in the opposite direction results in a decrease of the chemoattractant. For a schematic summary of the image processing steps and the different modi of operation see Fig. 2.

\section{Microfluidics}

Our flow photolysis experiments are performed in microfluidic channels that offer a high level of control due to the strictly laminar flow conditions. These devices are fabricated using standard soft lithography techniques. ${ }^{9,21}$ First, a negative foto mask with the microchannel pattern is designed and printed on a transparent film (JD Photo-Tools). A silicon wafer is coated with a $40 \mu \mathrm{m}$ layer of SU-8 photoresist (micro resist technology $\mathrm{GmbH}$ ) using standard spin coating and soft baking protocols. The coated wafer is then exposed to UV light using a Tamarack UV-Exposure Unit model PRX 500/1000/2000. The film mask is placed between the UV light source and the wafer to initiate cross-linking of the photoresist only in those areas that are part of the channel structures. Finally, the wafer is placed in a developer solution of acetone and isopropyl alcohol (VWR). The developer dissolves the non-crosslinked parts of the photoresist, resulting in a wafer that carries the desired channel layout as a photoresist microstructure (so-called master wafer).

The structured wafer serves as a mold for the fabrication of the microfluidic chip by standard soft lithography. A $5 \mathrm{~mm}$ layer of a mixture of PDMS and curing agent (Sylgard) is 


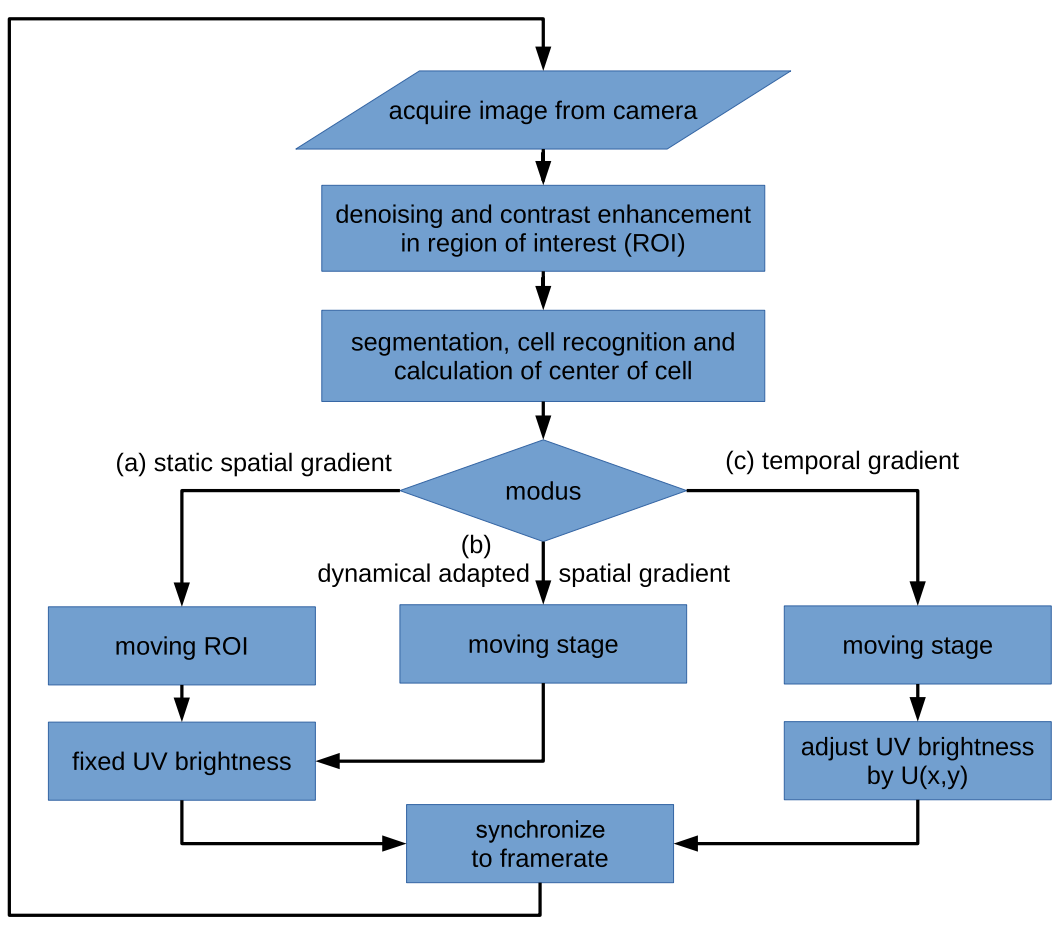

FIG. 2. Flow chart summarizing the successive steps of image processing and the different modi of operation of the setup.

given onto the wafer, air bubbles are removed under reduced pressure inside a desiccator, and the PDMS is hardened by baking at $80^{\circ} \mathrm{C}$ for $180 \mathrm{~min}$. After baking, the PDMS is cut and detached from the master wafer. Inlets and outlets are punched into the PDMS with a syringe needle. Finally, the device is sealed from below with a glass cover slip. This is achieved by a 3 min treatment of the PDMS block and the glass slide in an air plasma inside an PDC002 plasma cleaner (Harrick Plasma). When brought into contact immediately after plasma treatment, a permanent bond is formed between the PDMS block and the glass cover slip. The microfluidic channels used in our experiments were $40 \mu \mathrm{m} \times 1500 \mu \mathrm{m} \times 35 \mathrm{~mm}$ in size.

Prior to the experiment, a suspension of starvation developed Dictyostelium cells is infused into the microfluidic channel, and the cells are allowed to settle down and attach to the glass bottom surface for approximately $15 \mathrm{~min}$. Then, a $0.25 \mathrm{ml}$ glass syringe (Hamilton Bonaduz) is filled with Sørensen buffer containing $10 \mu \mathrm{M}$ of DMNB-caged cAMP, ${ }^{25}$ mounted onto a PHD 2000 syringe pump (Harvard Apparatus), and connected to the other inlet of the microchannel via a polytetrafluoroethylene (PTFE) tubing $(0.56 \mathrm{~mm} \times 1.07 \mathrm{~mm}$, Adtech Polymer Engineering). The solution is infused at a volume rate of $0.15 \mu \mathrm{l} / \mathrm{min}$ resulting in a constant flow speed of $41 \mu \mathrm{m} / \mathrm{s}$ inside the channel.

\section{Preparation of cell cultures}

For our realtime tracking experiments Dictyostelium discoideum WT-AX2 cells have been used. The cells were grown as an adherent culture in polystyrene cell culture flasks (Sarstedt AG \& Co) at $21{ }^{\circ} \mathrm{C}$ in HL5 medium (Formedium, Ltd.). For the preparation of an experiment, cells are washed twice and starved in shaking Sørensen buffer $\left(2 \mathrm{~g} \mathrm{KH}_{2} \mathrm{PO}_{4}\right.$, $0.36 \mathrm{~g} \mathrm{Na}_{2} \mathrm{HPO}_{4} \cdot 2 \mathrm{H}_{2} \mathrm{O}$ per $11, \mathrm{pH} 6.0$ ) with a density of about
$6.5 \cdot 10^{6}$ cells $/ \mathrm{ml}$. To synchronize development, cells were pulsed with $50 \mathrm{nM}$ cAMP every $6 \mathrm{~min}$, so that the cAMP concentration increased by that value with every pulse. When cells start to aggregate after about $6 \mathrm{~h}$, part of the cell suspension was sucked through a PTFE tubing into a disposable syringe. The tubing was then connected to an inlet of the microchannel and the suspension was infused into the channel.

\section{THEORETICAL ESTIMATE OF THE CHEMOATTRACTANT PROFILE}

In order to determine the chemoattractant concentration profile at the location of the cell inside the microfluidic device, we perform numerical simulations of the underlying physical model. It contains the photolytic release of cAMP and the advective and diffusive transport in the microchannel with a given flow profile.

\section{A. Flow profile in the microchannel}

For an incompressible Newtonian fluid under stationary flow conditions and at a low Reynolds number, the time dependent Navier-Stokes equation simplifies to a time independent Poisson equation. For a channel with a rectangular cross section, constant fluid volume flow, and no-slip boundary conditions, the full solution of this equation yields a velocity profile along the channel height and width that can be expressed by an infinite sum of harmonics. ${ }^{26}$ If the channel has infinite width, i.e., for a unidirectional flow between two infinitely extending parallel plates, the solution simplifies to a parabolic profile along the channel height.

In the case of a large but finite channel width, i.e., when the width is much larger than the channel height, deviations from the parabolic profile are observed. However, when measured far away from the side walls, the velocity profile in 


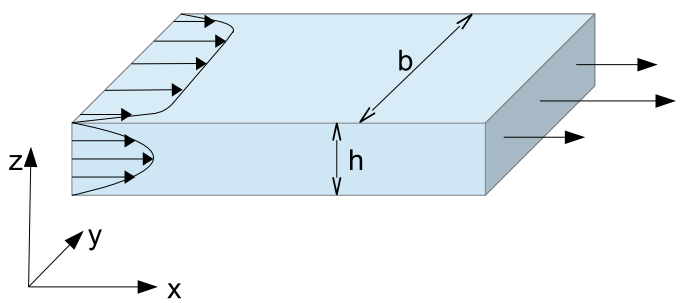

FIG. 3. Representation of the microfluidic channel with definition of the coordinate axes. The channel width and height are denoted by $b$ and $h$, respectively. Fluid flow runs from left to right.

the $z$-direction can be still approximated by the solution of the infinitely wide channel with an additional correction factor $\kappa$,

$$
\vec{v}=\frac{6 \kappa \dot{V}}{h^{3} b} z(h-z) \vec{e}_{x},
$$

where $\dot{V}$ denotes the volume inflow rate, $b$ is the width, $h$ the height of the channel, and $\vec{e}_{x}$ the unit vector in $x$-direction, see also Fig. 3. The correction factor $\kappa$ is determined by comparing the velocity profile for infinitely extending parallel plates with the solution for a channel of finite width. As the volume flow is directly proportional to the average cross-sectional flow velocity, the effect of the correction factor is to increase the speed in the central region of the channel to compensate for the lower volume flow at the side walls,

$$
\kappa=\frac{\left\langle v_{\infty}\right\rangle}{\langle v\rangle}=\frac{2}{3} \frac{v_{\max }}{\langle v\rangle},
$$

where $\left\langle v_{\infty}\right\rangle$ denotes the mean velocity of a flow between two infinitely extending parallel plates. Numerator and denominator are infinite series. Transforming this equation into a symmetric form that depends on $h / b$ and $b / h$ yields a suitable parameter $\vartheta$ to fit Equation (2). Determining the coefficients leads to an approximation within a maximum error of $0.2 \%$,

$$
\kappa \approx 1+0.792 \vartheta-0.0113 \sin (4 \pi \vartheta), \quad \vartheta=\left(\frac{h}{b}+\frac{b}{h}\right)^{-1} .
$$

\section{B. Intensity of the uncaging light}

To reliably calculate the amount of released cAMP, a precise estimate of the uncaging light intensity inside the microfluidic channel is required. In particular, we need to take the losses in light intensity due to reflection and absorption into account that occur when the light passes the interfaces between different media. The reflection loss of a perpendicular entry from one into another medium can be derived from the Fresnel equations,

$$
R_{12}=\left(\frac{n_{2}-n_{1}}{n_{2}+n_{1}}\right)^{2} .
$$

When the beam passes the glass slide it experiences losses due to reflections $R_{\mathrm{ag}}$ upon entering the slide, losses due to absorption within the glass slide itself $A_{\mathrm{g}}=1-\mathrm{e}^{-\alpha_{\mathrm{g}} \Delta z} \approx \alpha_{\mathrm{g}} \Delta z$, and losses $R_{\mathrm{gs}}$ due to reflections when passing the second surface between glass and solution. Here, $R_{\text {ag }}$ denotes the reflectance of the air/glass interface, $A_{\mathrm{g}}$ the absorbance of the glass with absorption coefficient $\alpha_{\mathrm{g}}$, and $R_{\mathrm{gs}}$ the reflectance of the glass/solution interface. Neglecting second order reflections and low absorption of the aqueous solution inside the channel, the effective transmission $T$ is approximately

$$
T=\left(1-R_{\mathrm{ag}}\right)\left(1-A_{\mathrm{g}}\right)\left(1-R_{\mathrm{gs}}\right) .
$$

For the setup used here we obtain $T=0.94$. Once the beam with an initial power density $S_{0}(x, y)$ has passed the different interfaces, only an amount of $S(x, y)=T S_{0}(x, y)$ is available within the channel.

\section{Light-induced release of CAMP}

If the solution that carries the caged cAMP is illuminated by UV light of wavelength $\lambda$, it absorbs energy per volume with a rate of

$$
\frac{\mathrm{d} E_{a}}{\mathrm{~d} A \mathrm{~d} t}=S(x, y) \alpha \mathrm{d} z .
$$

In general, the absorption coefficient $\alpha$ is different for different substances and wavelengths $\lambda$. This absorption coefficient can be derived from the extinction coefficient $\varepsilon$ and the concentration of the caged compound $c_{0}$ by $\alpha=\varepsilon c_{0} \ln 10$. Here, experimental values of $\varepsilon$ for aqueous solutions of the caged compound are used ${ }^{22}$ see Table II. Hydrolysis of the two isometric forms of DMNB-caged cAMP was taken into account for the time between the dissolution of the caged compound in water (both isomers in a ratio of 1:1) and the beginning of the experiment. During this time, they undergo hydrolysis at different rates, see Table II. The concentration $c_{0}$ is then given by the sum of the concentrations of both isomers at the beginning of the experiment. For the extinction coefficient $\varepsilon$ we take the average of the extinction coefficients of the two isomers, weighted by the corresponding concentration ratio. We do not consider thermal heating since the absorption of UV light in water can be neglected over the channel height. The absorbed energy $E_{a}$ corresponds to the number of absorbed photons $N_{a}$

$$
\mathrm{d} E_{a}=\frac{h c}{\lambda} \mathrm{d} N_{a} .
$$

Neglecting thermal heating, the absorbed energy splits molecules of the caged compound with a certain probability $\varphi$, denoted as the quantum yield, which corresponds to the number of chemoattractant molecules $N_{1}$ released per absorbed photon. With Eqs. (6) and (7) we obtain

$$
S(x, y) \alpha \varphi=\frac{h c}{\lambda} \frac{\mathrm{d} N_{1}}{\mathrm{~d} V \mathrm{~d} t} .
$$

We denote the concentration of the released signaling substance with $c_{1}$. Based on the relation between number density and concentration $\mathrm{d} N_{1} / \mathrm{d} V=N_{A} c_{1}$ with $N_{A}$ denoting the Avogadro constant, Eq. (8) can be rewritten as

$$
\frac{\mathrm{d} c_{1}}{\mathrm{~d} t}=\frac{\lambda \varphi \alpha S(x, y)}{h c N_{A}},
$$

which describes the temporal change in the chemoattractant concentration due to light-induced release by photo-uncaging.

\section{Spatiotemporal evolution of cAMP concentration}

The full spatiotemporal evolution of the concentrations of caged cAMP $\left(c_{0}\right)$ and released cAMP $\left(c_{1}\right)$ is governed by the 
respective reaction-diffusion-advection equations. Note that caged compounds are typically not stable in aqueous solution and degrade with a certain rate constant $k$. Together with the light-induced release described above, this decay is part of the source and sink terms that couple the equation for $c_{0}$ and $c_{1}$,

$$
\begin{aligned}
& \frac{\partial c_{0}}{\partial t}=\nabla\left(D_{0} \nabla c_{0}\right)-\vec{v} \cdot \nabla c_{0}-\left(k+\frac{\lambda \varphi \varepsilon \ln 10}{h c N_{A}} S\right) c_{0}, \\
& \frac{\partial c_{1}}{\partial t}=\nabla\left(D_{1} \nabla c_{1}\right)-\vec{v} \cdot \nabla c_{1}+\left(k+\frac{\lambda \varphi \varepsilon \ln 10}{h c N_{A}} S\right) c_{0} .
\end{aligned}
$$

Here, $D_{0}$ and $D_{1}$ denote the diffusion constants of caged and released cAMP, respectively. If diffusive transport of a substance with diffusivity $D$ along the height of the channel is much faster than advective transport, the concentration profile along the height levels out and a two dimensional calculation is sufficient. This approximation holds if the Taylor condition is fulfilled, ${ }^{19,32,33}$

$$
\frac{h^{2}}{4 \pi^{2} D} \ll \frac{l}{\langle v\rangle},
$$

which compares the typical diffusion time with the time scale of advective transport over the distance $l$ between the uncaging region and the position of the cell in the channel. For the twodimensional approximation in a rectangular channel, TaylorAris theory yields an effective diffusion coefficient in flow direction of ${ }^{32-35}$

$$
D_{\|}=D+\frac{\langle v\rangle^{2} h^{2}}{210 D},
$$

while the diffusion coefficient $D$ perpendicular to the flow remains unchanged. Thus, $D_{0}$ and $D_{1}$ in Eqs. (10) and (11) are two-dimensional matrices of the form

$$
\underline{D}=\left(\begin{array}{cc}
D_{\|} & 0 \\
0 & D
\end{array}\right) \text {. }
$$

\section{E. Numerical integration}

We solved the system of Equations (10) and (11) numerically using a finite difference scheme. To save CPU time, we relied on the two-dimensional approximation based on Taylor dispersion as explained above. The images taken by the microscope camera displayed an area of $470 \times 350 \mu \mathrm{m}^{2}$. For the integration of Eqs. (10) and (11) this area was discretized with an equidistant grid with spatial step size $\chi_{x}=\chi_{y}=3 \mu \mathrm{m}$.
To avoid boundary effects, the grid was extended for $50 \mu \mathrm{m}$ beyond the region of interest. The maximum time step $\tau$ per iteration was derived by the von Neumann stability analysis. For a two dimensional grid we obtained

$$
\tau \leq \min \left(\left(\frac{2 D_{\|}}{\chi_{x}^{2}}+\frac{2 D}{\chi_{y}^{2}}\right)^{-1}, \frac{2 D_{\|}}{v_{x}^{2}}\right) .
$$

To compute the light-induced cAMP release, the space dependent power density $S_{0}(x, y)$ is required. It was derived experimentally from a measurement of the total power of the uncaging light beam and a grayscale fluorescence image of the illuminated uncaging area. The latter was obtained in an independent experiment, where a fluorescein solution was filled into the microfluidic channel and excited with the uncaging light source. The experimentally derived profile of $S_{0}(x, y)$ is shown in Fig. 4(a) and was used in our numerical simulations. Based on the experimentally recorded trajectories, cAMP concentration and CAMP gradient were computed at every cell position for all time steps.

\section{F. Approximations and uncertainty}

We assumed that the light intensity is only a function of $x, y$, and $t$. As UV light absorption over the $40 \mu \mathrm{m}$ channel height in $z$-direction is very low, the resulting error is negligible. With the Taylor condition fulfilled, we could also reduce the flow and transport equations to a two-dimensional form. We neglected the local distortions of the velocity field induced by the cell itself. However, considering the flow speed in our chamber and the diffusivity of cAMP, this is a valid approximation. $^{16}$

To estimate errors in the numerically derived cAMP concentration profiles that were introduced by the discretization scheme of our simulations, we have performed worst case test runs, with a grid size and time step close to the limit of numerical stability. In these cases, the maximum error was about $10 \%$, which can be seen as an upper limit of the error introduced by our simulations. Similarly, we have compared the results of two- and three-dimensional simulations. Here, the maximum difference was on the order of only a few percent.

An additional uncertainty stems from the values of model parameters that were extracted from the literature, in particular, the diffusion coefficients $D$ of the caged and uncaged forms of the chemoattractant, the quantum yield $\varphi$, and the extinction coefficients $\varepsilon$. In addition, if the caged chemoattractant is prone to hydrolysis, the initial concentrations (a)

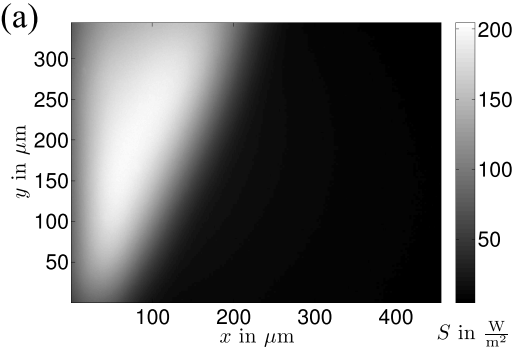

(b)

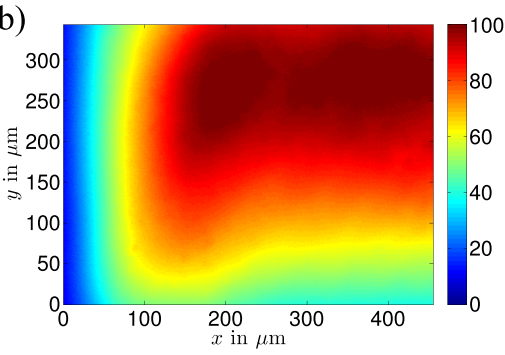

FIG. 4. (a) Spatial distribution of UV power intensity for triangular uncaging region in $\mathrm{W} / \mathrm{m}^{2}$. (b) Relative fluorescence intensity of fluorescein released by photolysis from a $50 \mu \mathrm{M}$ solution of CMNB-caged fluorescein under steady flow conditions. Fluid flows from left to right with $41 \mu \mathrm{m} / \mathrm{s}$, uncaging region as shown in (a). 

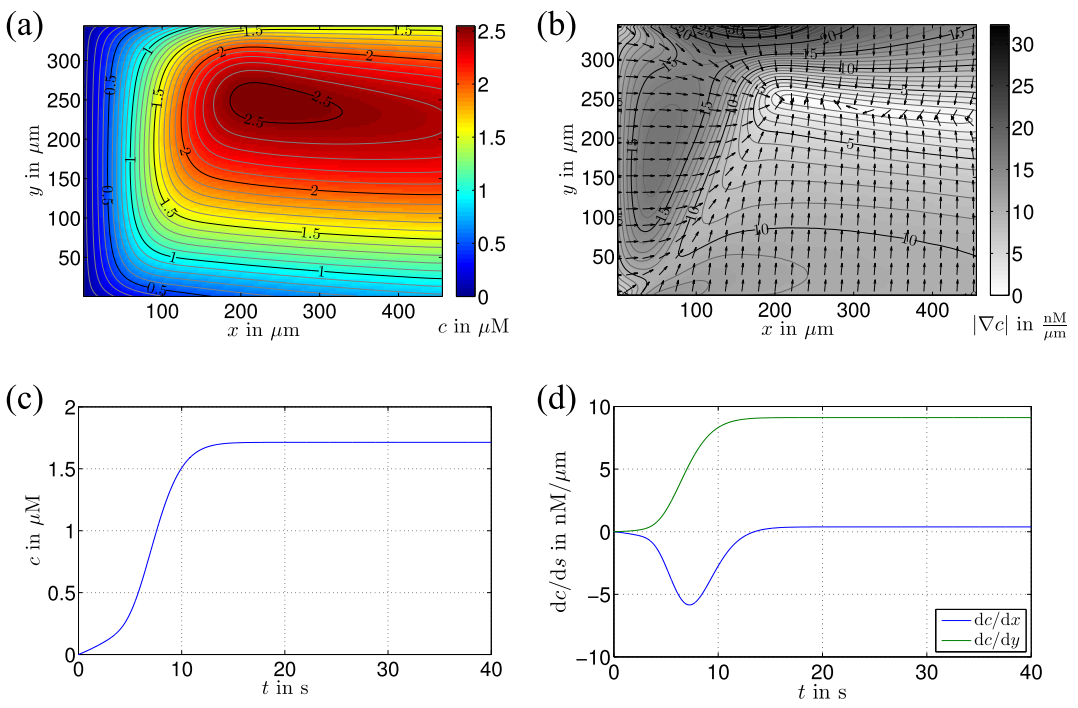

FIG. 5. (a) Numerical simulation of the steady state concentration field and (b) its absolute gradient and gradient direction for a flow speed of $41 \mu \mathrm{m} / \mathrm{s}$ of a $10 \mu \mathrm{M}$ DMNB-cAMP solution with the UV illumination profile shown in Fig. 4(a). (c) Saturation of concentration and (d) of its gradient components at the position of the cell, $x=350 \mu \mathrm{m}$ and $y=150 \mu \mathrm{m}$, after switching on the UV uncaging light source. $c_{0}$ and $c_{1}$ depend on the corresponding decay time. Here, we rely on the error estimates of the corresponding literature sources, see the references given in Table II.

\section{RESULTS}

This section is divided into two parts. First, we will characterize the adaptive microfluidic gradient generator. Second, we will compare chemotaxis of the social amoeba Dictyostelium discoideum under two different gradient conditions, (a) a static spatial cAMP gradient and (b) a dynamically adapted spatial cAMP gradient.

\section{A. Characterizing the setup}

All experiments are performed inside a microfluidic channel and viewed under an optical microscope as described in Sec. II. The channel is oriented such that fluid with the caged compound flows from left to right through the field of view. Light emitted by a UV-LED with a wave length of $\lambda=365 \mathrm{~nm}$ is directed through a triangular aperture and coupled into the light path of the microscope, such that a triangular region within the field of view is exposed to the UV light as shown in Fig. 4(a). The exposure time of the caged compound to the UV light and thus the amount of photochemically released substance depends on the flow speed and the width of the illuminated region in flow direction. For a triangular uncaging region, the width and thus the amount of released substance change perpendicular to the flow direction. In this way, the triangular geometry of the uncaging region is converted into a concentration gradient perpendicular to the flow direction downstream of the illuminated area. $^{18}$

Here, we will perform chemotaxis experiments with the social amoeba Dictyostelium discoideum. They require a caged version of the chemoattractant cAMP. However, as cAMP cannot be directly imaged, we rely on an uncaging experiment with CMNB-caged fluorescein to characterize the performance of our setup. With the help of CMNB-caged fluorescein, we can visualize the spatiotemporal concentration profile downstream of the uncaging region by fluorescence excitation of the uncaged fluorescein, see Fig. 4(b). The fluorescence distribution can be taken as an estimate for the relative shape of the cAMP concentration profile, as fluorescein and cAMP have similar diffusion coefficients. However, the absolute value of the cAMP concentration cannot be measured in this way.

For a quantitative estimate, we rely on the theoretical approach to calculate the concentration of photo-chemically released cAMP that was described in Sec. III. In particular, we perform numerical simulations of Equations (10) and (11) to determine the absolute cAMP concentration and its gradient at every point in space and time depending on the power of the UV light source. Figure 5(a) shows the stationary concentration field after the initial transient as determined by numerical simulations. The simulation parameters are given in Tables I and II. Together with the experimentally measured cell trajectories, the numerical simulation yields the cAMP concentration and the concentration gradient which each cell is experiencing over time. Based on this information, several parameters to characterize the chemotactic behaviour can be determined.

We also characterized the initial transient in the concentration profile that is observed after switching on the uncaging light source. To illustrate how the concentration profile is established, the temporal evolution of the concentration and of its gradient is shown in Figs. 5(c) and 5(d) at a fixed location downstream of the uncaging region $(x=350 \mu \mathrm{m}$, $y=150 \mu \mathrm{m})$. For the present choice of parameters, the switching time is about $10 \mathrm{~s}$. Note that during the switching, also a

TABLE I. Optophysical properties of the different materials in the optical pathway of the microfluidic device used here for an illumination wavelength of $\lambda=365 \mathrm{~nm}$.

\begin{tabular}{|c|c|c|}
\hline Medium & Refraction index $n$ & Absorption coefficient $\alpha$ in $10^{-3} \mathrm{~cm}^{-1}$ \\
\hline Air & 1.00 & $\approx 0$ \\
\hline D263 glass $^{27}$ & 1.52 & 708 \\
\hline Water $^{28}$ & 1.35 & 1.6181 \\
\hline PDMS $^{29,30}$ & 1.40 & $<51$ \\
\hline
\end{tabular}


TABLE II. Diffusive and optical properties of different caged compounds in aqueous solution.

\begin{tabular}{lccccc}
\hline \hline & $\begin{array}{c}\text { Extinction } \\
\text { coefficient } \\
\varepsilon \text { in } \mathrm{M}^{-1} \mathrm{~cm}^{-1}\end{array}$ & $\begin{array}{c}\text { Quantum } \\
\text { yield } \\
\varphi\end{array}$ & $\begin{array}{c}\text { Absorption } \\
\text { maximum } \\
\lambda \text { in nm }\end{array}$ & $\begin{array}{c}\text { Hydrolysis } \\
\text { decay time } \\
\tau \text { in } 10^{3} \mathrm{~s}\end{array}$ & $\begin{array}{c}\text { Diffusion } \\
\text { coefficient } \\
D \text { in } \mu \mathrm{m}^{2} / \mathrm{s}\end{array}$ \\
\hline DMNB-cAMP ax. $^{22}$ & 5800 & 0.05 & 345 & 125 & 267 \\
DMNB-cAMP equ. $^{22}$ & 6000 & 0.05 & 346 & 20.8 & 267 \\
CMNB-fluorescein $^{31}$ & 16500 & 0.13 & 334 & Stable & \\
cAMP $^{31}$ & $\ldots$ & $\ldots$ & $\ldots$ & Stable & 440 \\
Fluorescein & $\ldots$ & $\ldots$ & $\ldots$ & Stable & 425 \\
\hline \hline
\end{tabular}

transient gradient in the direction of the uncaging light source occurs.

In its current configuration, our setup can track cells with a speed of up to $20 \mu \mathrm{m} / \mathrm{s}$. This limit is imposed by the cutoff parameter of the particle tracking algorithm and the maximal frame rate in our experiments. When increasing the frame rate, the critical speed could be increased at the cost of lower contrast and resolution. However, most adherent eukaryotic cells move much slower than this critical speed, so that the current settings are sufficient for most applications. When tracking motile cells that are much slower, the only constraint is that the stability of the caged compound has to be compatible with the duration of the experiment. In particular, during the experiment, the concentration of the caged material should not change by more than a few percent. This can be estimated from the hydrolysis decay time of the caged compound.

\section{B. Dictyostelium chemotaxis in static and dynamically adapted gradients}

We now compare the chemotactic performance of Dictyostelium cells under two different gradient conditions. (a) In a static gradient, the concentration profile remains constant in space, corresponding to a fixed position of the source of chemoattractant. Here, the average concentration in the vicinity of the cell increases as the cell moves up the gradient, while the gradient remains constant in case of a linear profile. (b) In a dynamically adapted gradient, cell movement is compensated by automatically adjusting the position of the microscope stage. Thus, the moving cell remains at the same position in the gradient profile. In this way, both the average concentration and the slope in the concentration profile are kept constant at the position of the cell.

In Fig. 6, two sets of cell trajectories are shown that were recorded under conditions (a) and (b), respectively.
Visual inspection does not indicate any qualitative differences between the two data sets. In both cases, directed cell movement along the concentration gradient (in the positive $\mathrm{y}$-direction) is observed on average. In addition, cells have a tendency to move upstream (in the negative $\mathrm{x}$-direction). This may be induced either by the transient gradient in flow direction following initiation of the uncaging process, see Fig. 5(d), or it may be related to the mechanical stimulation of the cells by fluid shear stress (rheotaxis). ${ }^{36}$

To illustrate the difference between the two types of gradient experiments performed here, we display the time traces of the cAMP concentration and the cAMP gradient at the positions of individual cells for both types of experiments. In Figs. 7(a) and 7(b), we show the temporal evolution of concentration and gradient steepness for cells that move in a static gradient. It can be clearly seen, how the concentration increases over time as some of the cells move up the gradient. Also the gradient steepness shows a slight time dependence indicating that the gradient profile is not strictly linear. In Figs. 7(c) and 7(d), the corresponding time traces for the dynamically adapted gradient are shown for comparison. Here both the concentration and its gradient remain constant as the movement of cells is compensated by the microscope stage so that each cell remains at a fixed position in the gradient profile.

In Table III, we show the results of a quantitative cell track analysis for the two cases and a control data set acquired with the same setup but in the absence of a cAMP gradient. The average cAMP concentration $\langle c\rangle$ and the average cAMP gradient $\langle|\nabla c|\rangle$ the cells are experiencing are similar in both gradient cases. Also, the overall gradient direction is the same, $\left\langle\left|\phi_{\nabla c}\right|\right\rangle \sim 90^{\circ}$. The average speed $\langle v\rangle$ is clearly increased in the presence of a cAMP gradient compared to the control. However, also here no difference between the two gradient cases is observed. (a)

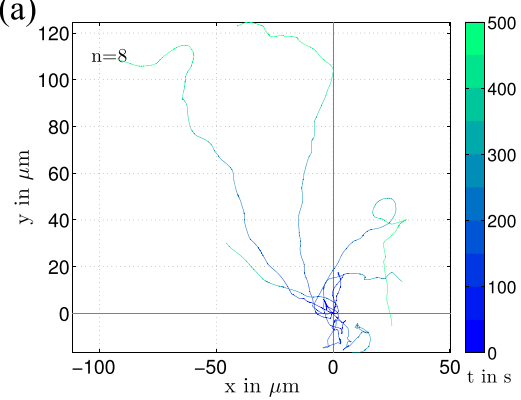

(b)

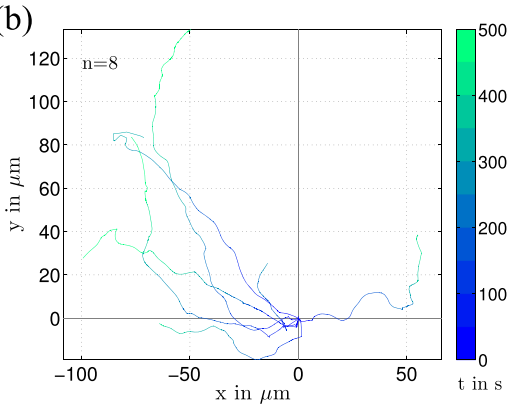

FIG. 6. Trajectories of chemotactic Dictyostelium cells (a) in a static spatial cAMP gradient and (b) in a dynamically adapted cAMP gradient. The starting points of all trajectories are shifted to the origin; colors are coding the time after starting the measurement. 
(a)

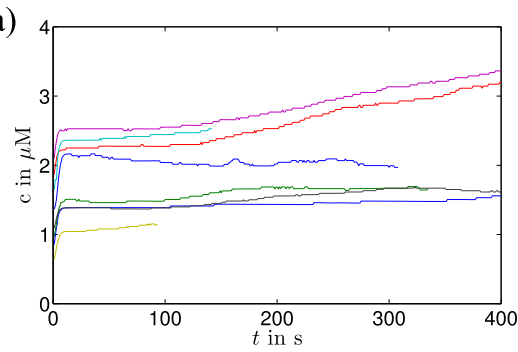

(c)

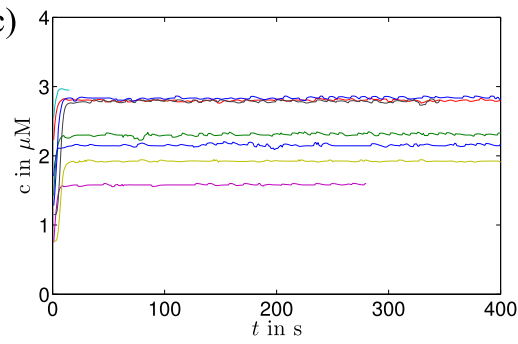

(b)

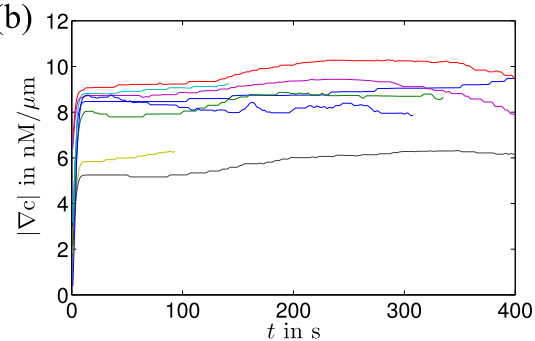

(d)

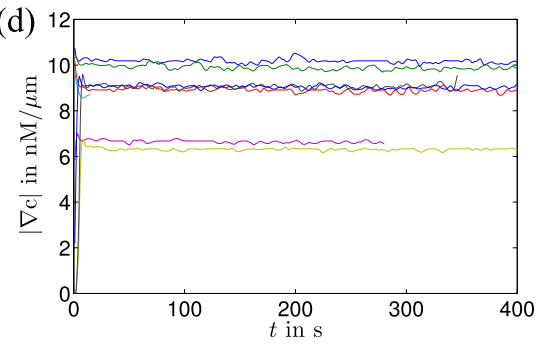

FIG. 7. (a) Concentration and (b) its gradient determined by numerical simulation at the position of Dictyostelium cells moving in a static spatial cAMP gradient. (c) Concentration and (d) its gradient determined by numerical simulation at the position of cells moving in a dynamically adapted cAMP gradient.

TABLE III. Comparison of chemotactic parameters' errors is given as standard error, angles are given in degree.

\begin{tabular}{lrrrccccc}
\hline \hline mode & \multicolumn{1}{c}{$\begin{array}{c}\langle v\rangle \\
(\mu \mathrm{m} / \mathrm{min})\end{array}$} & $\left\langle\phi_{v}\right\rangle(\mathrm{deg})$ & $\begin{array}{c}\langle c\rangle \\
(\mu \mathrm{M})\end{array}$ & $\begin{array}{c}\langle|\nabla c|\rangle \\
(\mathrm{nM} / \mu \mathrm{m})\end{array}$ & $\left\langle\left|\phi_{\nabla c}\right|\right\rangle(\mathrm{deg})$. & $\begin{array}{c}C_{I} \\
\phi_{\text {ref }}=90^{\circ}\end{array}$ & $\begin{array}{c}\tilde{C}_{I} \\
\text { resp. to }\end{array}$ \\
\hline Control & 44 & $12.0 \pm 1.2$ & $199 \pm 13$ & 0 & 0 & $\ldots$ & $(0.11 \pm 0.05)$ & $\ldots$ \\
Natural & 8 & $16.8 \pm 2.4$ & $93 \pm 25$ & $2.23 \pm 0.28$ & $8.03 \pm 0.52$ & $89.8 \pm 1.4$ & $0.54 \pm 0.12$ & $0.53 \pm 0.12$ \\
Adaptive & 8 & $16.8 \pm 1.8$ & $129 \pm 24$ & $2.35 \pm 0.15$ & $8.59 \pm 0.51$ & $92.7 \pm 0.3$ & $0.48 \pm 0.09$ & $0.50 \pm 0.08$ \\
\hline \hline
\end{tabular}

To quantify the directionality of cell movement, we determined the chemotactic index $C_{I}$ a common parameter that measures the chemotactic performance of a cell. It is typically defined as the distance traveled in gradient direction (the positive y-direction in our case) divided by the total path length. For a constant speed this definition is equal to the mean of $\cos \phi$ taken along the entire trajectory, where $\phi$ is the angle between the velocity vector and the gradient direction. The control experiment displays a chemotactic index close to zero, indicating that in the absence of a cAMP gradient, the probability for a cell to move in the positive and negative ydirections is equal. In contrast, the chemotactic index is clearly positive in both gradient cases, where similar $C_{I}$ values were observed. We note, however, that the $C_{I}$ is slightly higher in the case of a static gradient even though the average gradient was lower in this case. This indicates that chemotaxis is more efficient in cases where the average chemoattractant concentration increases while the cell moves up the gradient. Thus, out of the two cases tested here, cells perform better in the situation that is more frequently encountered under natural conditions, i.e., in the presence of a spatially fixed source of chemoattractant.

Note that the time resolved character of our numerical simulations also allows for the definition of an alternative chemotactic index $\tilde{C}_{I}$. In contrast to the chemotactic index $C_{I}$ that is defined with respect to a fixed spatial direction, $\tilde{C}_{I}$ is based on the local and possibly time dependent gradient direction. While this does not significantly change our present results (see Table III), this alternative definition may be use- ful if more sophisticated time-dependent stimulation protocols are used.

We have also compared the chemotactic performance in a static spatial gradient with results from conventional microfluidic gradient chambers and found good agreement (data not shown). In particular, for steep cAMP gradients, saturation of the chemotactic response occurred at similar gradient values and agreed with estimates of the receptor occupancy based on the known $K_{d}$ value of the Dictyostelium cAMP receptor. ${ }^{37}$

\section{CONCLUSION}

In summary, we have presented an adaptive microfluidic gradient generator for chemotactic single cell stimulation. Our setup combines the local generation of chemoattractant stimuli by photo-uncaging in a micro-flow (flow photolysis) with a real-time cell tracker that is coupled to a motorized microscope stage, so that the chemoattractant stimulus can be adapted in response to cell movement. In a first set of experiments, we exemplified our technique by comparing data from cells that move in a static spatial gradient with cells that move in a dynamically adapted gradient, where both the average local concentration and the gradient steepness are kept constant at the position of the moving cell.

We envision numerous future applications for our setup. For example, our setup will allow us to study the role of temporal versus spatial sensing in eukaryotic chemotaxis. For swimming bacteria that typically move in run-and-turn 
patterns, it is well know that they respond to chemoattractant gradients by modulating their turning rate in response to temporal changes of the chemoattractant concentration (temporal sensing). In contrast, many eukaryotic cells can measure spatial concentration gradients across their cell body even if they remain at a fixed location (spatial sensing). However, also in the case of eukaryotic chemotaxis, additional contributions from a temporal sensing mechanism may play a role. Our adaptive single cell stimulation setup opens up ways to study such mechanisms. In particular, we can stimulate chemotactic cells with a spatially uniform chemoattractant concentration that changes depending on the direction of motion of the cells as explained in Sec. II B, scenario (c). Only cells that are able to perform temporal sensing will be able to identify the direction of motion, which is rewarded by an increasing uniform chemoattractant concentration.

\section{ACKNOWLEDGMENTS}

We thank Kirsten Sachse for continuous help with the Dictyostelium cell culture. Financial support by the Deutsche Forschungsgemeinschaft is gratefully acknowledged (Grant No. BE 3978/3-1 to CB).

${ }^{1}$ A. J. Ridley, M. A. Schwartz, K. Burridge, R. A. Firtel, M. H. Ginsberg, G. Borisy, J. Thomas Parsons, and A. R. Horwitz, "Cell migration: Integrating signals from front to back," Science 302(5651), 1704-1709 (2003).

${ }^{2}$ T. M. Keenan and A. Folch, "Biomolecular gradients in cell culture systems," Lab Chip 8(1), 34-57 (2007).

${ }^{3}$ D. Zicha, G. A. Dunn, and A. F. Brown, "A new direct-viewing chemotaxis chamber," J. Cell Sci. 99(4), 769-775 (1991).

${ }^{4} \mathrm{~S}$. Boyden, "The chemotactic effect of mixtures of antibody and antigen on polymorphonuclear leucocytes," J. Exp. Med. 115(3), 453-466 (1962).

${ }^{5} \mathrm{~S}$. H. Zigmond, "Ability of polymorphonuclear leukocytes to orient in gradients of chemotactic factors," J. Cell Biol. 75(2), 606-616 (1977).

${ }^{6}$ A. J. Muinonen-Martin, D. M. Veltman, G. Kalna, and R. H. Insall, "An improved chamber for direct visualisation of chemotaxis," PLoS One 5(12), e15309 (2010).

${ }^{7}$ P. Suraneni, B. Rubinstein, J. R. Unruh, M. Durnin, D. Hanein, and R. Li, "The Arp $2 / 3$ complex is required for lamellipodia extension and directional fibroblast cell migration," J. Cell Biol. 197, 239 (2012).

${ }^{8}$ D. N. Breslauer, P. J. Lee, and L. P. Lee, "Microfluidics-based systems biology," Mol. BioSyst. 2(2), 97-112 (2006).

${ }^{9}$ D. C. Duffy et al., "Rapid prototyping of microfluidic systems in poly(dimethylsiloxane)," Anal. Chem. 70(23), 4974-4984 (1998).

${ }^{10} \mathrm{~S}$. Kim, H. Joon Kim, and N. L. Jeon, "Biological applications of microfluidic gradient devices," Integr. Biol. 2(11-12), 584-603 (2010).

${ }^{11} \mathrm{C}$. Beta and E. Bodenschatz, "Microfluidic tools for quantitative studies of eukaryotic chemotaxis," Eur. J. Cell Biol. 90(10), 811-816 (2011).

${ }^{12}$ A. G. G. Toh, Z. P. Wang, C. Yang, and N.-T. Nguyen, "Engineering microfluidic concentration gradient generators for biological applications," Microfluid. Nanofluid. 16(1-2), 1-18 (2013).

${ }^{13}$ N. Li Jeon, S. K. W. Dertinger, D. T. Chiu, I. S. Choi, A. D. Stroock, and G. M. Whitesides, "Generation of solution and surface gradients using microfluidic systems," Langmuir 16(22), 8311-8316 (2000).

${ }^{14}$ D. L. Englert, M. D. Manson, and A. Jayaraman, "Flow-based microfluidic device for quantifying bacterial chemotaxis in stable, competing gradients," Appl. Environ. Microbiol. 75(13), 4557-4564 (2009).
${ }^{15}$ T. Ahmed, T. S. Shimizu, and R. Stocker, "Microfluidics for bacterial chemotaxis," Integr. Biol. 2(11-12), 604-629 (2010).

${ }^{16}$ C. Beta, T. Fröhlich, H. U. Bödeker, and E. Bodenschatz, "Chemotaxis in microfluidic devices-A study of flow effects," Lab Chip 8(7), 1087-1096 (2008).

${ }^{17}$ M. Etzrodt, H. C. F. Ishikawa, J. Dalous, A. Müller-Taubenberger, T. Bretschneider, and G. Gerisch, "Time-resolved responses to chemoattractant, characteristic of the front and tail of Dictyostelium cells," FEBS Lett. 580(28-29), 6707-6713 (2006).

${ }^{18}$ C. Beta, D. Wyatt, W.-J. Rappel, and E. Bodenschatz, "Flow photolysis for spatiotemporal stimulation of single cells," Anal. Chem. 79(10), 3940-3944 (2007).

${ }^{19}$ A. J. Bae, C. Beta, and E. Bodenschatz, "Rapid switching of chemical signals in microfluidic devices," Lab Chip 9(21), 3059-3065 (2009).

${ }^{20}$ C. Westendorf, J. Negrete, A. J. Bae, R. Sandmann, E. Bodenschatz, and C. Beta, "Actin cytoskeleton of chemotactic amoebae operates close to the onset of oscillations," Proc. Natl. Acad. Sci. U. S. A. 110(10), 3853-3858 (2013).

${ }^{21}$ C. Beta, "Spatiotemporal stimulation of single cells using flow photolysis," in Chemotaxis, edited by T. Jin and D. Hereld (Humana Press, Totowa, NJ, 2009), Vol. 571, pp. 321-332.

${ }^{22}$ R. Givend and M. Goeldner, Dynamic Studies in Biology: Phototriggers, Photoswitches and Caged Biomolecules (WILEY-VCH Verlag GmbH \& Co. KGaA, Weinheim, 2005), pp. 159-163.

${ }^{23}$ J. S. Lim, Two-Dimensional Signal and Image Processing (Prentice Hall, Englewood Cliffs, NJ, 1990), p. 584.

${ }^{24}$ A. Anielski, E. K. B. Pfannes, and C. Beta, "Cell shape recognition and segmentation in fluorescence microscopy images," J. Comput. Interdiscip. Sci. 3(1-2), 99-106 (2012).

${ }^{25}$ J. M. Nerbonne, S. Richard, J. Nargeot, and H. A. Lester, "New photoactivatable cyclic nucleotides produce intracellular jumps in cyclic AMP and cyclic GMP concentrations," Nature 310(5972), 74-76 (1984).

${ }^{26} \mathrm{M}$. Spiga and G. L. Morino, "A symmetric solution for velocity profile in laminar flow through rectangular ducts," Int. Commun. Heat Mass Transfer 21(4), 469-475 (1994).

${ }^{27}$ P. K. Whitman, M. Staggs, C. W. Carr, S. Dixit, W. Sell, and D. Milam, "Performance of thin borosilicate glass sheets at 351-nm," in Annual Symposium on Optical Materials for High Power Lasers, 2001.

${ }^{28}$ G. M. Hale and M. R. Querry, "Optical constants of water in the $200-\mathrm{nm}$ to $200-\mu \mathrm{m}$ wavelength region," Appl. Opt. 12(3), 555-563 (1973).

${ }^{29}$ J. E. Mark, Polymer Data Handbook (Oxford University Press, 1999), pp. 411-435.

${ }^{30}$ M.-H. Wu, K. E. Paul, and G. M. Whitesides, "Patterning flood illumination with microlens arrays," Appl. Opt. 41(13), 2575-2585 (2002).

${ }^{31}$ M. Dworkin and K. H. Keller, "Solubility and diffusion coefficient of adenosine 3': 5'-monophosphate," J. Biol. Chem. 252(3), 864-865 (1977).

${ }^{32} \mathrm{G}$. Taylor, "Dispersion of soluble matter in solvent flowing slowly through a tube," Proc. R. Soc. A 219(1137), 186-203 (1953).

${ }^{33} \mathrm{R}$. Aris, "On the dispersion of a solute in a fluid flowing through a tube," Proc. R. Soc. A 235(1200), 67-77 (1956).

${ }^{34}$ D. A. Beard, "Taylor dispersion of a solute in a microfluidic channel," J. Appl. Phys. 89(8), 4667-4669 (2001).

${ }^{35}$ K. D. Dorfman and H. Brenner, "Comment on 'Taylor dispersion of a solute in a microfluidic channel' [J. Appl. Phys. 89, 4667 (2001)],” J. Appl. Phys. 90(12), 6553-6554 (2001).

${ }^{36}$ J. Dalous, E. Burghardt, A. Müller-Taubenberger, F. Bruckert, G. Gerisch, and T. Bretschneider, "Reversal of cell polarity and actin-myosin cytoskeleton reorganization under mechanical and chemical stimulation," Biophys. J. 94(3), 1063-1074 (2008).

${ }^{37}$ L. Song, S. M. Nadkarni, H. U. Bödeker, C. Beta, A. Bae, C. Franck, W.-J. Rappel, W. F. Loomis, and E. Bodenschatz, "Dictyostelium discoideum chemotaxis: Threshold for directed motion,” Eur. J. Cell Biol. 85, 981-989 (2006). 